\title{
Exploring hyperhidrosis and related thermoregulatory symptoms as a possible clinical identifier for the dysautonomic subtype of Parkinson's disease
}

\author{
Daniel J. van Wamelen ${ }^{1,2,3}$ (1) Valentina Leta ${ }^{1,2} \cdot$ Aleksandra M. Podlewska $^{1,2} \cdot$ Yi-Min Wan $^{1,2} \cdot$ Katarina Krbot $^{1,2,4}$. \\ Elina Jaakkola ${ }^{5} \cdot$ Pablo Martinez-Martin ${ }^{6} \cdot$ Alexandra Rizos $^{1,2} \cdot$ Miriam Parry $^{1,2} \cdot$ Vinod Metta $^{2}$. \\ Kallol Ray Chaudhuri ${ }^{1,2}$
}

Received: 29 January 2019 / Revised: 1 April 2019 / Accepted: 12 April 2019 / Published online: 17 April 2019

(c) The Author(s) 2019

\begin{abstract}
Objective To identify associated (non-)motor profiles of Parkinson's disease (PD) patients with hyperhidrosis as a dominant problem.

Methods This is a cross-sectional, exploratory, analysis of participants enrolled in the Non-motor Longitudinal International Study (NILS; UKCRN No: 10084) at the Parkinson's Centre at King's College Hospital (London, UK). Hyperhidrosis scores (yes/no) on question 28 of the Non-Motor Symptom Questionnaire were used to classify patients with normal sweat function $(n=172)$ and excessive sweating $(n=56)$ (Analysis $1 ; n=228)$. NMS scale (NMSS) question 30 scores were used to stratify participants based on hyperhidrosis severity (Analysis $2 ; n=352$ ) using an arbitrary severity grading: absent score 0 $(n=267)$, mild 1-4 $(n=49)$, moderate 5-8 $(n=17)$, and severe 9-12 $(n=19)$. NMS burden, as well as PD sleep scale (PDSS) scores were then analysed along with other correlates.

Results No differences were observed in baseline demographics between groups in either analysis. Patients with hyperhidrosis exhibited significantly higher total NMSS burden compared to those without $(p<0.001)$. Secondary analyses revealed higher dyskinesia scores, worse quality of life and PDSS scores, and higher anxiety and depression levels in hyperhidrosis patients $(p<0.001)$. Tertiary analyses revealed higher NMSS item scores for fatigue, sleep initiation, restless legs, urinary urgency, and unexplained pain $(p<0.001)$.

Conclusions Chronic hyperhidrosis appears to be associated with a dysautonomia dominant subtype in PD patients, which is also associated with sleep disorders and a higher rate of dyskinesia (fluctuation-related hyperhidrosis). These data should prompt the concept of hyperhidrosis being used as a simple clinical screening tool to identify PD patients with autonomic symptoms.
\end{abstract}

Keywords Parkinson's disease $\cdot$ Hyperhidrosis $\cdot$ Autonomic $\cdot$ Non-motor symptoms $\cdot$ Dyskinesia

Daniel J. van Wamelen

Daniel.van_Wamelen@kcl.ac.uk

1 King's College London, Institute of Psychiatry, Psychology and Neuroscience, Department of Basic and Clinical Neurosciences, De Crespigny Park, London SE5 8AF, UK

2 Parkinson Foundation Centre of Excellence, King's College Hospital, Denmark Hill, London SE5 9RS, UK

3 Radboud University Medical Centre, Department of Neurology, Donders Institute for Brain, Cognition and Behaviour, Nijmegen, The Netherlands
4 Klinik fur Gerontopsychiatrie, Asklepios Nord-Ochsenzoll, Hamburg, Germany

5 Division of Clinical Neurosciences, University of Turku, Turku, Finland

6 National Centre of Epidemiology and CIBERNED, Carlos III Institute of Health, Madrid, Spain 


\section{Introduction}

Hyperhidrosis is one of the least studied non-motor symptoms (NMS) in Parkinson's disease (PD). Sweating disorders in PD include mainly hyperhidrosis, but also hypohydrosis, and the prevalence is reported by some at $64 \%$ of PD patients, compared to $12.5 \%$ of healthy controls [1]. Phenomenologically, hyperhidrosis in PD could be chronic or paroxysmal, on the one hand, and non-fluctuating or related to non-motor fluctuations, on the other [2]. The phenomenon of hyperhidrosis may also overlap with an increase of sebum excretion rate known as seborrhoea which is also frequent in PD patients [3]. The pathophysiology remains unclear but dysautonomia has been suggested as a possible correlate, while sweating occurs both during levodopa-induced motor fluctuations [4] and dyskinesia [5]. The pattern of dyshidrosis in PD appears to differ from the general population and axial hyperhidrosis in PD is associated with decreased activation of sweat glands in the palms of the hands suggesting that axial hyperhidrosis could be a compensatory phenomenon for reduced sympathetic function in the extremities [5].

Recently, Sauerbier et al. and Marras and Chaudhuri $[6,7]$ proposed several non-motor subtypes in PD, analogues to the better-known motor subtypes. One of these phenotypes is an autonomic phenotype, with adrenergic/ cholinergic features (such as orthostatic hypotension) and gastro-intestinal symptoms overlapping with sleep dysfunction and levodopa-induced dyskinesias [6-8]. Here, we investigate, using validated tools (Non-Motor Questionnaire and Scale), whether in PD patients with selfdeclared hyperhidrosis specific patterns of the autonomic subtype of PD exist.

\section{Methods}

This is a post hoc, exploratory study using the Non-motor International Longitudinal Study's (NILS) international database. We used data from patients clinically diagnosed with idiopathic PD and whose data were entered between November 2011 and September 2018. We only included the baseline assessments (of five yearly longitudinal assessments) of patients seen at King's College Hospital London (NHS Foundation Trust). The NILS Study is the world's first non-motor focussed comprehensive longitudinal cohort study addressing non-motor profiling of PD and the natural history of non-motor symptoms together with treatment response and clinico-pathological correlations. The study is adopted as a national study by the National Institute of Health Research in the UK (UKCRN No:
10084) and involves 14 centres across Europe, but for the current study only patients who had a baseline assessment at King's College Hospital were included. The study was authorised by local ethics committees (NRES SouthEast London REC3, 10084, 10/H0808/141). All patients gave written consent prior to study procedures in accordance with the Declaration of Helsinki. Exclusion criteria were (1) diagnosis of Parkinsonism different to idiopathic PD; (2) dementia (as per internationally accepted criteria); and (3) inability for giving consent to participate in the study.

Data extracted from the NILS database concerned sex, disease onset and duration (in years), and Levodopa equivalent dose (LEDD). Patient-reported outcomes were: Hospital Anxiety and Depression Scale (HADS; a 14-item, patient-completed scale with subscales for anxiety and depression) [10]; PDQ-8 (a specific instrument for assessment of health-related quality of life in PD) [11]; PD Sleep Scale-version 1 (PDSS), a 15-item, patientcompleted clinical tool used to assess the frequency of sleep disturbances during the past week in PD patients) [12], and Epworth Sleepiness Scale (ESS) scores (6-item tool looking at daytime sleepiness) [13]. Clinician-based evaluations were: Hoehn and Yahr (HY) staging [9] and Non-Motor Symptoms Scale (NMSS). The NMSS considers the frequency and severity of the non-motor symptoms of PD grouped into nine domains: cardiovascular, sleep/ fatigue, mood/apathy, perceptual problems/hallucinations, attention/memory, gastrointestinal tract, urinary function, sexual function, and miscellaneous $[14,15]$.

Hyperhidrosis scores on question 28 of the validated Non-Motor Symptom Questionnaire (NMSQ; when available) were used to classify patients with normal sweat function $(n=172)$ and hyperhidrosis $(n=56)$ (analysis 1 ). In addition, we used the NMSS question 30 scores to stratify participants based on hyperhidrosis severity (analysis 2): absent score $0(n=267)$, mild scores $1-4$ $(n=49)$, moderate scores 5-8 $(n=17)$, and severe scores 9-12 $(n=19)$. Subsequently, we assessed differences in NMS burden measured on the NMSS. Secondary and tertiary outcomes included differences in specific non-motor (HADS, PDSS, ESS) and motor scores. As this was an exploratory analysis, we did not perform cluster analysis. Group differences were tested with Mann-Whitney $U$ test or Kruskal-Wallis test, where appropriate. A Bonferroni correction was used to correct for multiple testing for the secondary $(p=0.05 / 8)$ and tertiary $(p=0.05 / 30)$ outcomes. To test for gender differences, Pearson Chi-square analysis was used. For correlations, Spearman's analysis was used. All data were analysed using SPSS Version 24 (IBM SPSS Statistics for Windows, Version 24.0. Armonk, NY: IBM Corp.). 


\section{Results}

No differences were observed in age, gender, disease duration, and Levodopa Equivalent Dose (LED) between patients with and without hyperhidrosis (analysis 1). There were also no baseline differences in the NMSS defined groups (analysis 2), except a trend for LED ( $p=0.017$; Table 1). 160 of the $172(93 \%)$ patients who indicated no sweating on the NMSQ had no hyperhidrosis on the NMSS scale (absent score) confirming the high concordance between the NMSQ and NMSS.

In both analyses, patients with hyperhidrosis exhibited significantly higher NMSS total scores (analysis 1: 73.4 vs $41.6 ; p<0.001$ and analysis $2: 41.9$ for absent, 58.2 for mild, 77.8 for moderate, and 93.7 for severe; $p<0.001$ ) (Fig. 1; Tables 1 and 2). Secondary analyses revealed, after correction for multiple testing, significantly higher Scales for Outcomes in PD (SCOPA) dyskinesia scores in hyperhidrosis patients ( $p<0.001$ for analysis 1 and $p=0.001$ for analysis 2 ), worse quality of life scores (PDQ-8; $p<0.001$ both analyses), worse PDSS ( $p=0.006$ for analysis 1 and $p<0.001$ analysis 2$)$ and higher anxiety levels $(p<0.001$ for analysis 1 and $p=0.003$ for analysis 2 ) and depression levels $(p<0.001$ for analysis 1$)$ on the Hospital Anxiety and Depression Scale (Tables 1 and 2). In addition, we correlated hyperhidrosis severity (defined by NMSS item 30) with SCOPA dyskinesia scores, and also with SCOPA fluctuation presence and severity subitem scores. The analysis showed a significant positive correlation between hyperhidrosis severity and dyskinesia (for both SCOPA dyskinesia total scores, and subitems 20 and 21 for fluctuation presence and severity; rho $\geq 0.162 ; p \leq 0.002$ ).

Tertiary analyses, corrected for multiple testing, revealed higher NMSS scores for restless legs, urinary urgency and pain (analysis 1; $p<0.001$; Table 1) and for fatigue, sleep initiation, and restless legs (analysis $2 ; p<0.001$ ), with nearly significant trends for urinary frequency, pain and anosmia (analysis $2 ; p=0.004$; Table 2). Moreover, there were significant positive correlations between hyperhidrosis severity and fatigue, sleep initiation, restless legs, hyposmia, and weight change (rho $\geq 0.177 ; p<0.001$ ), with statistical trends for daytime sleepiness, depression, and urinary urgency (rho $\geq 0.158$; $p=0.003$ ).

\section{Discussion}

In this exploratory study, we observed a significantly higher NMS burden in PD patients with hyperhidrosis, especially among those with the most severe hyperhidrosis.
Table 1 Demographics and (non-)motor profiles (non-motor symptom questionnaire based) associated with hyperhidrosis in Parkinson's disease

\begin{tabular}{|c|c|c|c|}
\hline \multicolumn{4}{|l|}{ Analysis 1 (NMSQ item 28) } \\
\hline Hyperhidrosis & No $(n=172)$ & Yes $(n=56)$ & $P$ \\
\hline \multicolumn{4}{|l|}{ Demographics } \\
\hline Age (years) & $64.7 \pm 11.4$ & $62.6 \pm 10.8$ & 0.20 \\
\hline Gender (M/F) & $119 / 53$ & $36 / 20$ & 0.50 \\
\hline Disease duration (years) & $5.0 \pm 4.9$ & $5.7 \pm 4.7$ & 0.30 \\
\hline $\operatorname{LED}(\mathrm{mg})$ & $522.9 \pm 440.0$ & $663.5 \pm 545.5$ & 0.09 \\
\hline NMSS total & $41.6 \pm 33.0$ & $73.4 \pm 48.8$ & $<0.001$ \\
\hline 1. Dizziness & $1.2 \pm 2.2$ & $1.9 \pm 2.8$ & 0.08 \\
\hline 2. Falls & $0.3 \pm 1.2$ & $0.8 \pm 2.1$ & 0.019 \\
\hline 3. Somnolence & $2.0 \pm 3.0$ & $2.8 \pm 3.9$ & 0.15 \\
\hline 4. Fatigue & $2.6 \pm 3.1$ & $4.4 \pm 4.4$ & 0.009 \\
\hline 5. Sleep initiation & $2.7 \pm 3.7$ & $4.3 \pm 4.5$ & 0.020 \\
\hline 6. Restless legs & $1.5 \pm 2.7$ & $3.0 \pm 3.8$ & 0.001 \\
\hline 7. Loss of interest & $1.1 \pm 2.5$ & $2.0 \pm 3.5$ & 0.10 \\
\hline 8. Motivation & $1.2 \pm 2.5$ & $2.3 \pm 3.7$ & 0.19 \\
\hline 9. Feeling nervous & $1.1 \pm 2.7$ & $2.9 \pm 3.8$ & 0.005 \\
\hline 10. Depression & $1.7 \pm 2.8$ & $3.2 \pm 3.8$ & 0.002 \\
\hline 11. Flat moods & $1.3 \pm 2.3$ & $1.6 \pm 2.8$ & 0.64 \\
\hline 12. Anhedonism & $1.1 \pm 2.4$ & $1.6 \pm 3.3$ & 0.64 \\
\hline 13. Hallucinations & $0.5 \pm 1.4$ & $0.8 \pm 2.2$ & 0.42 \\
\hline 14. Delusions & $0.2 \pm 1.0$ & $0.4 \pm 1.6$ & 0.80 \\
\hline 15. Diplopia & $0.3 \pm 1.1$ & $0.9 \pm 2.6$ & 0.25 \\
\hline 16. Concentration & $1.3 \pm 2.3$ & $2.1 \pm 2.9$ & 0.049 \\
\hline 17. Recall & $1.5 \pm 2.5$ & $2.6 \pm 3.0$ & 0.006 \\
\hline 18. Forgetfulness & $1.3 \pm 2.3$ & $1.8 \pm 2.5$ & 0.17 \\
\hline 19. Sialorrhoea & $1.2 \pm 2.3$ & $2.2 \pm 3.3$ & 0.018 \\
\hline 20. Dysphagia & $0.7 \pm 1.8$ & $1.4 \pm 2.5$ & 0.013 \\
\hline 21. Constipation & $1.6 \pm 2.5$ & $3.1 \pm 4.2$ & 0.047 \\
\hline 22. Urgency & $2.4 \pm 3.5$ & $4.7 \pm 4.6$ & 0.001 \\
\hline 23. Frequency & $1.6 \pm 3.0$ & $2.9 \pm 3.9$ & 0.036 \\
\hline 24. Nocturia & $2.7 \pm 3.6$ & $3.2 \pm 4.2$ & 0.72 \\
\hline 25. Libido & $1.4 \pm 2.9$ & $1.9 \pm 3.3$ & 0.10 \\
\hline 26. Performing sex & $1.3 \pm 3.0$ & $1.3 \pm 2.9$ & 0.68 \\
\hline 27. Pain & $1.4 \pm 2.7$ & $3.7 \pm 4.2$ & $<0.001$ \\
\hline 28. Anosmia & $2.7 \pm 3.7$ & $4.1 \pm 4.7$ & 0.046 \\
\hline 29. Weight change & $0.7 \pm 2.0$ & $1.4 \pm 3.2$ & 0.28 \\
\hline 30. Hyperhidrosis & - & - & - \\
\hline PDSS & $109.7 \pm 27.2$ & $98.1 \pm 28.2$ & 0.006 \\
\hline ESS & $7.4 \pm 4.7$ & $9.0 \pm 6.0$ & 0.16 \\
\hline HADS anxiety & $5.3 \pm 3.8$ & $8.1 \pm 4.3$ & $<0.001$ \\
\hline HADS depression & $4.9 \pm 3.9$ & $7.0 \pm 4.1$ & $<0.001$ \\
\hline PDQ-8 & $7.1 \pm 5.4$ & $11.7 \pm 7.4$ & $<0.001$ \\
\hline SCOPA-motor & $10.3 \pm 5.0$ & $11.4 \pm 5.2$ & 0.13 \\
\hline SCOPA-daily living & $5.1 \pm 3.3$ & $6.8 \pm 4.4$ & 0.019 \\
\hline SCOPA-complications & $1.5 \pm 1.9$ & $3.0 \pm 3.3$ & 0.002 \\
\hline
\end{tabular}

Statistical differences tested using Mann-Whitney $U$ test $M$ male, $F$ female, $L E D$ Levodopa equivalent dose, PDSS Parkinson's disease sleep scale, ESS Epworth sleepiness scale, HADS hospital anxiety and depression scale, $P D Q-8$ 8-item Parkinson's disease quality of life scale, SCOPA Scales for outcomes in Parkinson's disease 


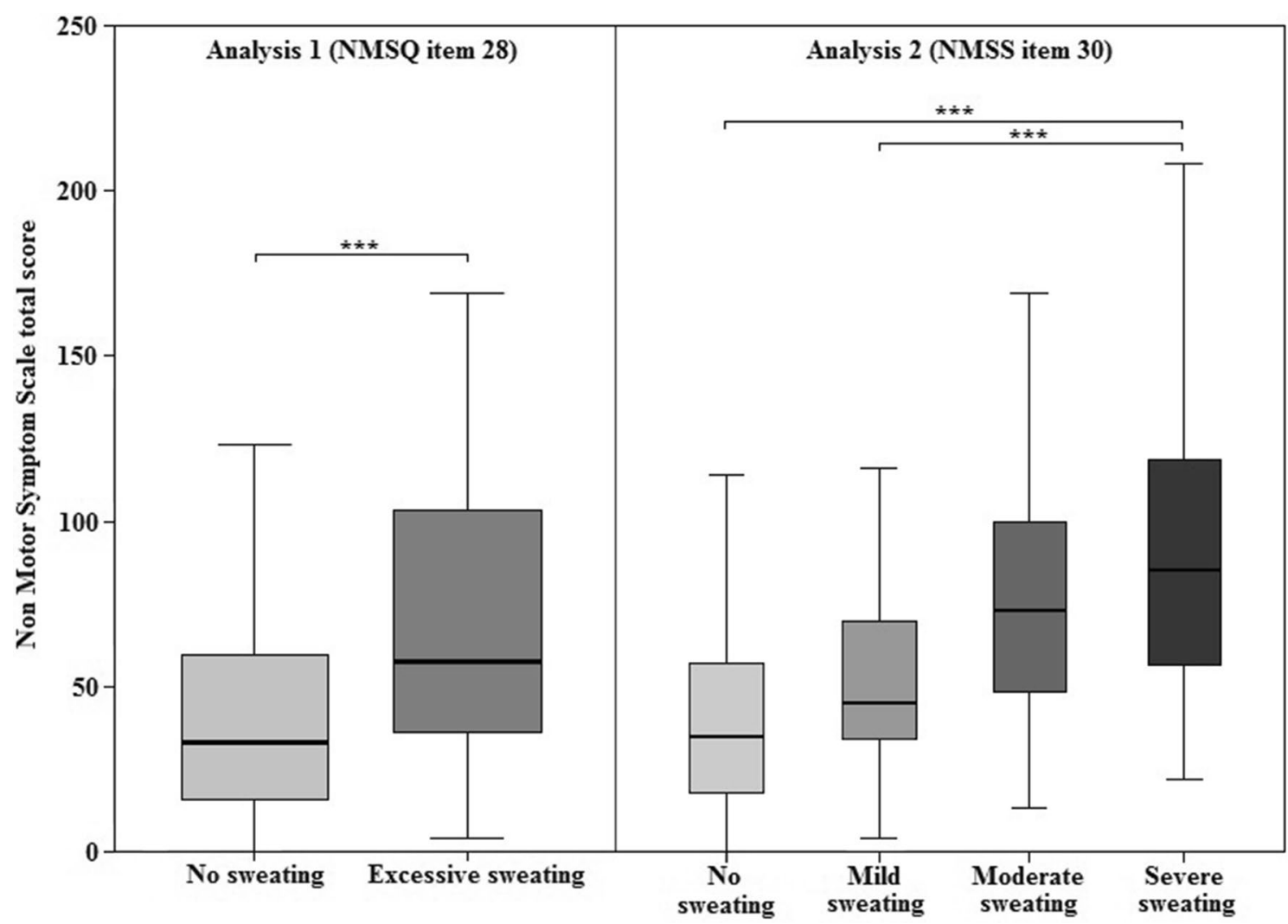

Fig. 1 Non-motor symptom Scale total scores differences between Parkinson's disease patients with hyperhidrosis and no hyperhidrosis and across the different severities of hyperhidrosis. NMSQ Non- motor symptom questionnaire, NMSS Non-motor symptom scale, $* * * p<0.001$. Statistical differences tested using Mann-Whitney $U$ and Kruskal-Wallis tests

strategies for PD. It has been shown that PD patients with autonomic dysfunction in general, but also individual autonomic abnormalities, such as sweating disorders, show a more rapid disease progression and shorter survival compared to those without such abnormalities [17]. Moreover, hyperhidrosis is associated with pain and decreased quality of life [16]. Whether treatment of autonomic symptoms and hyperhidrosis leads to slowing down of the disease progression and improvement of survival remains currently unclear. Moreover, due to the lack of validated and evidence-based algorithms, the treatment of hyperhidrosis in PD patients remains challenging, and its effectiveness limited.

The only motor difference between PD patients suffering from hyperhidrosis and those without was an increased severity of dyskinesia. It might be argued that, from a mechanistic point of view, dyskinesia itself leads to increased sweating. However, a recent meta-analysis on the risk of excessive sweating with antidepressants showed that hyperhidrosis is associated with increased dopamine receptor affinity of specific antidepressants [18], underlining the link between hyperhidrosis and dopamine metabolism. Other explanations could be found in the hypothalamus, one of the key structures in thermoregulation. It is known that the hypothalamus is affected by Lewy of the emerging concept of holistic personalised medicine 
Table 2 Demographics and (non-)motor profiles (nonmotor symptom scale based) associated with hyperhidrosis in Parkinson's disease

\begin{tabular}{|c|c|c|c|c|c|}
\hline \multicolumn{6}{|l|}{ Analysis 2 (NMSS item 30) } \\
\hline Hyperhidrosis & No $(n=267)$ & Mild $(n=49)$ & Moderate $(n=17)$ & Severe $(n=19)$ & $P$ \\
\hline \multicolumn{6}{|l|}{ Demographics } \\
\hline Age (years) & $64.5 \pm 11.6$ & $63.4 \pm 11.4$ & $60.8 \pm 11.4$ & $62.3 \pm 11.5$ & 0.61 \\
\hline Gender (M/F) & $183 / 84$ & $33 / 16$ & $12 / 5$ & $8 / 11$ & 0.13 \\
\hline Disease duration (years) & $5.2 \pm 5.1$ & $6.3 \pm 5.6$ & $4.2 \pm 3.2$ & $6.9 \pm 3.8$ & 0.033 \\
\hline LED (mg) & $522.7 \pm 467.2$ & $613.4 \pm 477.6$ & $631.5 \pm 473.8$ & $815.3 \pm 460.0$ & 0.017 \\
\hline NMSS total & $41.9 \pm 32.3$ & $58.2 \pm 39.6$ & $77.8 \pm 41.6$ & $93.7 \pm 52.6$ & $<0.001$ \\
\hline 1. Dizziness & $1.4 \pm 2.2$ & $1.5 \pm 2.5$ & $1.9 \pm 3.2$ & $2.7 \pm 3.3$ & 0.31 \\
\hline 2. Falls & $0.4 \pm 1.3$ & $0.4 \pm 1.2$ & $0.4 \pm 1.2$ & $1.4 \pm 3.0$ & 0.028 \\
\hline 3. Somnolence & $1.7 \pm 3.0$ & $2.4 \pm 3.1$ & $2.1 \pm 3.3$ & $3.9 \pm 4.2$ & 0.014 \\
\hline 4. Fatigue & $2.4 \pm 3.1$ & $4.2 \pm 3.9$ & $5.0 \pm 3.7$ & $6.1 \pm 4.6$ & $<0.001$ \\
\hline 5. Sleep initiation & $2.7 \pm 3.6$ & $3.5 \pm 3.6$ & $4.9 \pm 4.2$ & $6.9 \pm 4.1$ & $<0.001$ \\
\hline 6. Restless legs & $1.6 \pm 2.8$ & $1.8 \pm 2.0$ & $4.2 \pm 3.9$ & $3.7 \pm 3.8$ & $<0.001$ \\
\hline 7. Loss of interest & $1.1 \pm 2.4$ & $1.3 \pm 2.6$ & $3.4 \pm 4.1$ & $1.1 \pm 3.0$ & 0.31 \\
\hline 8. Motivation & $1.1 \pm 2.4$ & $1.8 \pm 2.8$ & $2.2 \pm 4.1$ & $2.1 \pm 3.5$ & 0.15 \\
\hline 9. Feeling nervous & $1.7 \pm 2.9$ & $2.3 \pm 3.1$ & $2.5 \pm 3.6$ & $3.7 \pm 4.5$ & 0.31 \\
\hline 10. Depression & $1.7 \pm 2.8$ & $2.7 \pm 3.3$ & $3.5 \pm 3.3$ & $2.5 \pm 3.9$ & 0.005 \\
\hline 11. Flat moods & $1.2 \pm 2.2$ & $1.7 \pm 2.7$ & $1.9 \pm 2.7$ & $1.7 \pm 3.2$ & 0.37 \\
\hline 12. Anhedonism & $0.9 \pm 2.2$ & $1.9 \pm 3.3$ & $1.4 \pm 3.0$ & $1.0 \pm 2.8$ & 0.008 \\
\hline 13. Hallucinations & $0.5 \pm 1.5$ & $0.5 \pm 1.3$ & $0.6 \pm 1.37$ & $1.6 \pm 3.9$ & 0.78 \\
\hline 14. Delusions & $0.2 \pm 0.8$ & $0.5 \pm 1.7$ & $0.6 \pm 1.7$ & $0.9 \pm 3.0$ & 0.86 \\
\hline 15. Diplopia & $0.4 \pm 1.4$ & $0.5 \pm 1.9$ & $1.3 \pm 3.4$ & $0.3 \pm 1.4$ & 0.66 \\
\hline 16. Concentration & $1.5 \pm 2.4$ & $1.7 \pm 2.6$ & $1.8 \pm 2.1$ & $2.8 \pm 4.1$ & 0.83 \\
\hline 17. Recall & $1.6 \pm 2.4$ & $2.1 \pm 2.7$ & $2.1 \pm 2.0$ & $3.2 \pm 3.8$ & 0.09 \\
\hline 18. Forgetfulness & $1.4 \pm 2.4$ & $1.7 \pm 2.6$ & $1.7 \pm 2.4$ & $2.1 \pm 2.9$ & 0.53 \\
\hline 19. Sialorrhoea & $1.1 \pm 2.4$ & $1.6 \pm 2.8$ & $0.8 \pm 2.0$ & $3.2 \pm 4.0$ & 0.049 \\
\hline 20. Dysphagia & $0.7 \pm 1.8$ & $1.4 \pm 2.8$ & $1.5 \pm 2.2$ & $0.6 \pm 1.1$ & 0.11 \\
\hline 21. Constipation & $1.9 \pm 2.9$ & $2.1 \pm 3.2$ & $1.9 \pm 3.2$ & $3.9 \pm 4.5$ & 0.05 \\
\hline 22. Urgency & $2.4 \pm 3.5$ & $3.4 \pm 4.2$ & $4.3 \pm 4.9$ & $4.8 \pm 4.6$ & 0.029 \\
\hline 23. Frequency & $1.7 \pm 2.9$ & $2.2 \pm 3.6$ & $1.9 \pm 4.0$ & $4.6 \pm 4.2$ & 0.003 \\
\hline 24. Nocturia & $2.6 \pm 3.4$ & $2.6 \pm 3.6$ & $3.5 \pm 4.2$ & $4.1 \pm 7.7$ & 0.28 \\
\hline 25. Libido & $1.3 \pm 2.7$ & $1.3 \pm 2.8$ & $3.4 \pm 4.4$ & $2.0 \pm 3.3$ & 0.68 \\
\hline 26. Performing sex & $1.2 \pm 2.8$ & $1.3 \pm 2.9$ & $2.3 \pm 4.4$ & $1.7 \pm 3.6$ & 0.21 \\
\hline 27. Pain & $2.0 \pm 3.2$ & $2.8 \pm 3.6$ & $4.7 \pm 4.7$ & $3.5 \pm 4.6$ & 0.004 \\
\hline 28. Anosmia & $2.8 \pm 3.8$ & $3.4 \pm 4.1$ & $5.4 \pm 4.4$ & $5.33 \pm 4.7$ & 0.004 \\
\hline 29. Weight change & $0.7 \pm 2.0$ & $1.0 \pm 2.0$ & $1.4 \pm 4.0$ & $2.3 \pm 4.2$ & 0.08 \\
\hline 30. Hyperhidrosis & - & - & - & - & - \\
\hline PDSS & $109.2 \pm 26.6$ & $95.1 \pm 31.1$ & $95.9 \pm 22.3$ & $89.4 \pm 23.0$ & $<0.001$ \\
\hline ESS & $7.4 \pm 4.8$ & $8.5 \pm 5.9$ & $8.9 \pm 6.0$ & $9.6 \pm 5.8$ & 0.44 \\
\hline HADS anxiety & $5.8 \pm 3.9$ & $7.6 \pm 3.8$ & $7.6 \pm 4.3$ & $8.4 \pm 5.1$ & 0.003 \\
\hline HADS depression & $5.3 \pm 3.7$ & $6.3 \pm 3.7$ & $7.5 \pm 5.0$ & $6.5 \pm 3.3$ & 0.043 \\
\hline PDQ-8 & $7.7 \pm 5.9$ & $11.1 \pm 6.6$ & $10.5 \pm 6.3$ & $9.6 \pm 5.8$ & $<0.001$ \\
\hline SCOPA-motor & $10.4 \pm 5.4$ & $11.5 \pm 6.5$ & $12.1 \pm 4.4$ & $13.9 \pm 6.5$ & 0.047 \\
\hline SCOPA-daily living & $5.5 \pm 3.7$ & $6.1 \pm 4.0$ & $6.5 \pm 4.1$ & $8.1 \pm 4.8$ & 0.08 \\
\hline SCOPA-complications & $1.7 \pm 2.2$ & $2.8 \pm 3.1$ & $2.1 \pm 1.7$ & $4.4 \pm 3.6$ & 0.001 \\
\hline
\end{tabular}

Statistical differences tested using Kruskal-Wallis test

$M$ male, $F$ female, $L E D$ Levodopa equivalent dose, NMSS non-motor symptom scale, PDSS Parkinson's disease sleep scale, ESS Epworth sleepiness scale, $H A D S$ hospital anxiety and depression scale, $P D Q-8$ 8 -item Parkinson's disease quality of life scale, SCOPA Scales for Outcomes in Parkinson's disease 
pathology in PD [19] and also the hypothalamic functional connectivity is disturbed in autonomic dysfunction in PD [20]. Furthermore, hyperhidrosis in PD is often episodic [18] and similar sweat attacks occur during menopause, where they have been related to brain noradrenergic over activity [21]. Perhaps, similar changes to the noradrenergic system occur in PD, explaining hyperhidrosis.

Limitations in this study include the cross-sectional design and retrospective data mining, rather than a longitudinal follow-up to assess the development of specific symptoms in PD patients suffering from hyperhidrosis. In addition, we did not assess symptoms related to impaired thermoregulation, and dehydration, often related to prominent dyskinesias [22]. Also, the presence and severity of hyperhidrosis were only assessed through history taking and not objectively assessed. Moreover, both NMSS and NMSQ contain only one item each addressing hyperhidrosis $[14,15]$. The NMSS and NMSQ are, however, validated tools for assessing NMS in PD patients and are commonly used tools in outpatient clinics, reflecting a real-world experience. As such we feel that our current results are useful, also considering that there were no group differences regarding disease duration, Hoehn and Yahr stages, LED and sex which in themselves can also influence NMS burden and dyskinesia severity.

In summary, the presence and severity of hyperhidrosis in PD patients coincides with an apparent PD endophenotype with dysautonomia which is associated with sleep disorders and a higher rate of dyskinesia. We suggest that asking about hyperhidrosis can be used as a simple screening tool to identify this specific subset of PD patients with dominant autonomic features, although further research is needed.

Acknowledgements We acknowledge data collection efforts by all contributors, collaborators, and administrative staff of the NILS study. This article presents independent research funded by the National Institute for Health Research (NIHR) Mental Health Biomedical Research Centre and Dementia Unit at South London and Maudsley NHS Foundation Trust and King's College London.

\section{Compliance with ethical standards}

Conflicts of interest On behalf of all authors, the corresponding author states that there is no conflict of interest.

Ethical approval The views expressed are those of the author(s) and not necessarily those of the NHS, the NIHR or the Department of Health. This research did not receive any specific grant from funding agencies in the public, commercial, or not-for-profit sectors.

Open Access This article is distributed under the terms of the Creative Commons Attribution 4.0 International License (http://creativeco mmons.org/licenses/by/4.0/), which permits unrestricted use, distribution, and reproduction in any medium, provided you give appropriate credit to the original author(s) and the source, provide a link to the Creative Commons license, and indicate if changes were made.

\section{References}

1. Schestatsky P, Valls-Solé J, Ehlers JA, Rieder CR, Gomes I (2006) Hyperhidrosis in Parkinson's disease. Mov Disord 21(10): $1744-1748$

2. Katunina E, Titova N (2017) The epidemiology of nonmotor symptoms in Parkinson's disease (cohort and other studies). Int Rev Neurobiol 133:91-110. https://doi.org/10.1016/ bs.irn.2017.05.012

3. Martignoni E, Godi L, Pacchetti C, Berardesca E, Vignoli GP, Albani G, Mancini F, Nappi G (1997) Is seborrhea a sign of autonomic impairment in Parkinson's disease? J Neural Transm (Vienna) 104(11-12):1295-1304

4. Storch A, Schneider CB, Wolz M, Stürwald Y, Nebe A, Odin P, Mahler A, Fuchs G, Jost WH, Chaudhuri KR, Koch R, Reichmann H, Ebersbach G (2003) Nonmotor fluctuations in Parkinson disease: severity and correlation with motor complications. Neurology 80(9):800-809

5. Swinn L, Schrag A, Viswanathan R, Bloem BR, Lees A, Quinn N (2003) Sweating dysfunction in Parkinson's disease. Mov Disord 18(12):1459-1463

6. Sauerbier A, Jenner P, Todorova A, Chaudhuri KR (2016) Non motor subtypes and Parkinson's disease. Parkinsonism Relat Disord 22(Suppl 1):S41-S46. https://doi.org/10.1016/j.parkreldis .2015.09.027

7. Marras C, Chaudhuri KR (2016) Nonmotor features of Parkinson's disease subtypes. Mov Disord 31(8):1095-1102. https://doi. org/10.1002/mds.26510

8. Titova N, Martinez-Martin P, Katunina E, Chaudhuri KR (2017) Advanced Parkinson's or “complex phase" Parkinson's disease? Re-evaluation is needed. J Neural Transm (Vienna) 124(12):15291537. https://doi.org/10.1007/s00702-017-1799-3

9. Hoehn MM, Yahr MD (1967) Parkinsonism: onset, progression and mortality. Neurology 17(5):427-442

10. Zigmond AS, Snaith RP (1983) The hospital anxiety and depression scale. Acta Psychiatr Scand 67:361-370

11. Jenkinson C, Fitzpatrick R, Peto V, Greenhall R, Hyman N (1997) The PDQ-development and validation of a short-form Parkinson's disease questionnaire. Psychol Health 12:805-814

12. Chaudhuri KR, Pal S, DiMarco A, Whately-Smith C, Bridgman K, Mathew R, Pezzela FR, Forbes A, Högl B, Trenkwalder C (2002) The Parkinson's disease sleep scale: a new instrument for assessing sleep and nocturnal disability in Parkinson's disease. J Neurol Neurosurg Psychiatry 73(6):629-635

13. Johns MW (1991) A new method for measuring daytime sleepiness: the Epworth sleepiness scale. Sleep 14(6):540-545

14. Martinez-Martin P, Rodriguez-Blazquez C, Abe K, Bhattacharyya KB, Bloem BR, Carod-Artal FJ, Prakash R, Esselink RA, Falup-Pecurariu C, Gallardo M, Mir P, Naidu Y, Nicoletti A, Sethi K, Tsuboi Y, van Hilten JJ, Visser M, Zappia M, Chaudhuri KR (2009) International study on the psychometric attributes of the non-motor symptoms scale in Parkinson disease. Neurology 73:1584-1591

15. Chaudhuri KR, Martinez-Martin P, Brown RG, Sethi K, Stocchi F, Odin P, Ondo W, Abe K, Macphee G, Macmahon D, Barone P, Rabey M, Forbes A, Breen K, Tluk S, Naidu Y, Olanow W, Williams AJ, Thomas S, Rye D, Tsuboi Y, Hand A, Schapira AH (2007) The metric properties of a novel non-motor symptoms scale for Parkinson's disease: results from an international pilot study. Mov Disord 22:1901-1911

16. Swinn L, Schrag A, Viswanathan R, Bloem BR, Lees A, Quinn N (2003) Sweating dysfunction in Parkinson's disease. Mov Disord 18(12):1459-1463 
17. de Pablo-Fernandez E, Tur C, Revesz T, Lees AJ, Holton JL, Warner TT (2017) Association of autonomic dysfunction with disease progression and survival in Parkinson disease. JAMA Neurol 74(8):970-976. https://doi.org/10.1001/jamaneurol.2017.1125

18. Coon EA, Low PA (2018) Thermoregulation in Parkinson disease. Handb Clin Neurol. 157:715-725

19. Langston JW, Forno LS (1978) The hypothalamus in Parkinson disease. Ann Neurol 3(2):129-133

20. Dayan E, Sklerov M, Browner N (2018) Disrupted hypothalamic functional connectivity in patients with $\mathrm{PD}$ and autonomic dysfunction. Neurology 90(23):e2051-e2058. https://doi. org/10.1212/WNL.0000000000005641

21. Freedman RR (2014) Menopausal hot flashes: mechanisms, endocrinology, treatment. J Steroid Biochem Mol Biol 142:115-120. https://doi.org/10.1016/j.jsbmb.2013.08.010

22. Sarchioto M, Ricchi V, Melis M, Deriu M, Arca R, Melis M, Morgante F, Cossu G (2018) Dyskinesia-hyperpyrexia syndrome in Parkinson's disease: A heat shock-related emergency? Mov Disord Clin Pract 5(5):534-537. https://doi.org/10.1002/mdc3.12663 